\title{
Flow of a thin liquid film coating a horizontal stationary cylinder
}

\author{
M. Cachile, ${ }^{*}$ M. A. Aguirre, M. Lenschen, and A. Calvo ${ }^{\dagger}$ \\ Grupo de Medios Porosos, Facultad Ingeniería, Universidad de Buenos Aires, Paseo Colón 850, 1063 Buenos Aires, Argentina
}

(Received 2 August 2012; revised manuscript received 26 June 2013; published 4 December 2013)

\begin{abstract}
An experimental and theoretical study of the flow of liquid films around a stationary horizontal cylinder is reported. The film presents two different behaviors: The flow is stable in the upper zone (up to $\sim 150^{\circ}$ with the vertical) and Rayleigh-Taylor-like instabilities appear in the lower zone. For the stable region, film thickness evolution could be described by numerically integrating an evolution equation obtained using a lubrication approximation. For the unstable region, a linear stability analysis allows us to determine the maximum growth wavelength for the Rayleigh-Taylor instability. Approximate analytical solutions were obtained for generatrices at an angle with the vertical $\theta=0$ (stable region) and $\theta=\pi$ (where the instability appears).
\end{abstract}

DOI: 10.1103/PhysRevE.88.063005

PACS number(s): 47.20.Ma, 68.15.+e

\section{INTRODUCTION}

The study of the flow of thin liquid films on a curved surface is of great interest in basic physics as well as for industries. In coating applications, such as corrosion protection or painting, the final uniformity and minimum thickness, among other factors, need to be characterized and controlled. For instance, in the design of heat exchangers, the characteristic times and distribution of flow around the solid are of crucial importance for the efficiency of the device. Many industrial coatings are based on products, usually liquids, that undertake phase transitions due to evaporation or polymerization (as in the case of paints or lacquers). The final distribution of the product mainly depends on the evolution of the film while it is in the liquid state [1] and the flow induced by the action of gravity and capillary effects modifies the initial film thickness distribution. In this way, instabilities associated with Rayleigh-Taylor instability appear at the lower part of the solids where the fluid is eventually thicker, introducing a new factor in the distribution of the films. This phenomenon produces a longer drying time in zones where the liquid is thicker, leading to parts on the solid where the film is dry and others where the drying is in progress.

In the literature there is no systematic experimental study of the thickness behavior for the conditions presented here, i.e., film thickness small compared to the cylinder curvature, despite the obvious interest in the subject. In contrast, there are several important works on the subject from the theoretical point of view [2,3]. Although simplified, the model developed in this work reproduces very well the experimental results, including the instability analysis.

In this work we study experimentally and theoretically the dynamics of a homogeneous film of liquid that coats a horizontal cylinder where the radius is much larger than the film thickness. The initial film thickness is prepared to be initially uniform, which is a usual condition in coating applications and also a natural initial condition for the theoretical study. From an academic point of view it is also interesting to study a geometrically simple system in which two different behaviors of the film coexist: a stable flow (in

*mcachil@fi.uba.ar

${ }^{\dagger}$ Deceased. an upper zone) and the density gradient between the film and the surrounding air favoring the onset of a Rayleigh-Taylor instability (in a lower zone).

The experimental setup and procedure are described in Sec. II. Experimental results obtained with nonvolatile Newtonian liquid are presented in Sec. III and a theoretical approach is presented in Sec. IV, in which the thickness evolution of the coating film will be described by numerical integration of the Navier-Stokes equations in the lubrication approximation [4]. Finally, results are analyzed and discussed in Sec. V.

\section{EXPERIMENTAL PROCEDURE}

The experimental setup is the same as that used in a previous work [5] and is shown schematically in Fig. 1. The cylinder is made of stainless steel and has a length $L=16 \mathrm{~cm}$ and diameter $D=15 \mathrm{~cm}$. The fluids used in the experiments, as in Ref. [5], are poly(dimethyl)siloxane (silicon oil) with viscosities $\mu=1000$ and $100 \mathrm{cp}$, density $\rho=0.97 \mathrm{~g} / \mathrm{cm}^{3}$, surface tension $\gamma=22.7 \mathrm{mN} / \mathrm{m}$, and the following very suitable characteristics: The fluid wets completely the surface and is nonvolatile. The former ensures that no dry spots will appear and the latter keeps constant the mass of the fluid and avoids the apparition of surface-tension gradients.

The initial uniform distribution of the liquid, which fully coats the cylinder, is obtained by pouring an appropriate volume of liquid on a calibrated distribution rod [1] kept in contact with the cylinder while turning at constant speed, as shown in Fig. 2. Once the liquid is uniformly distributed along the cylinder, the rod is removed and the cylinder stopped and the experiment begins. With this method, using different distribution rods, initial thickness values $H_{0}$ between 30 and $120 \mu \mathrm{m}$.

The optical displacement sensor (ODS) (shown in Fig. 1 from Acuity Research AR-600 [6]), which automatically displaces along the side of the frame where it is held, is used to determine the local thickness of the film coating the cylinder within $\pm 7 \mu \mathrm{m}$. The ODS is able to obtain around 2000 thickness measurements in each run along the cylinder length, which gives a precise thickness profile of a generatrix. The time lapse on each run $(\approx 15 \mathrm{~s})$ is short enough compared to the duration of an experiment (750-4500 s) so that the profile of the film $h(z, t)$ could be considered to be instantaneous. 


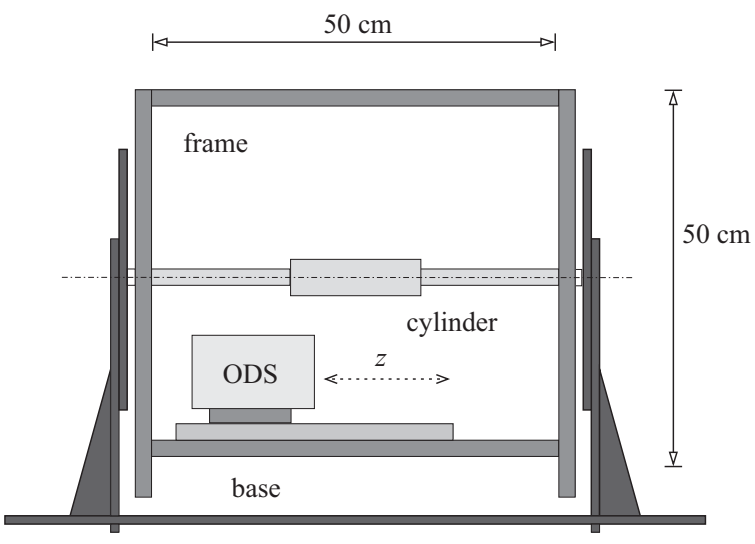

FIG. 1. Schematic view of the experimental setup used in the experiments. Here ODS denotes the optical displacement sensor.

The frame holding the ODS can be fixed in each experiment at the desired angle $\theta_{0}$ with respect to the vertical (see Fig. 2). The experiment begins once the cylinder is fully coated with a uniform film of thickness $H_{0}$. Seven values of $\theta_{0}$ were available: $\theta_{0}=n \pi / 6$ with $0 \leqslant n \leqslant 6$. Simultaneously with the ODS measurement, the lower generatrix is recorded with a video camera. Afterward images are processed and the temporal evolution is studied by means of spatiotemporal diagrams.

\section{EXPERIMENTAL RESULTS}

As mentioned before, the experiments are performed for angles of $0, \pi / 6, \pi / 3, \pi / 2,2 \pi / 3,5 \pi / 6$, and $\pi$ by coating the cylinder initially with a uniform film. Two different behaviors are observed in the film flow around the cylinder: While only a small region around the lower generatrix $(\theta \sim \pi)$ is unstable, the rest of the cylinder shows a stable behavior. Each zone is analyzed using different techniques.

\section{A. Stable region}

In this region $(\theta \lesssim \pi)$ the film thickness remains independent of $z$, at every measured profile, since the initial condition of the experiment is of uniform thickness throughout the cylinder. In this zone, the existence of the ends of the cylinder is apparent only from a small bump, which remains stationary

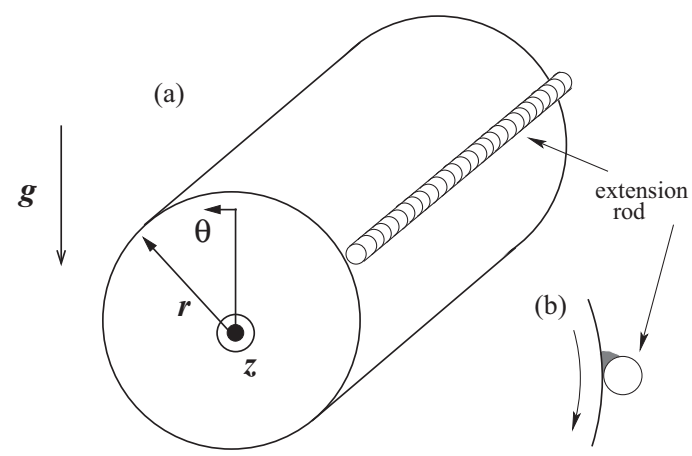

FIG. 2. Detail view of the experimental setup. (a) The extension rod is shown in position for the initial coating of the cylinder. (b) Detail of the contact zone between the extension rod and the cylinder while it rotates to form the initial uniform coating.

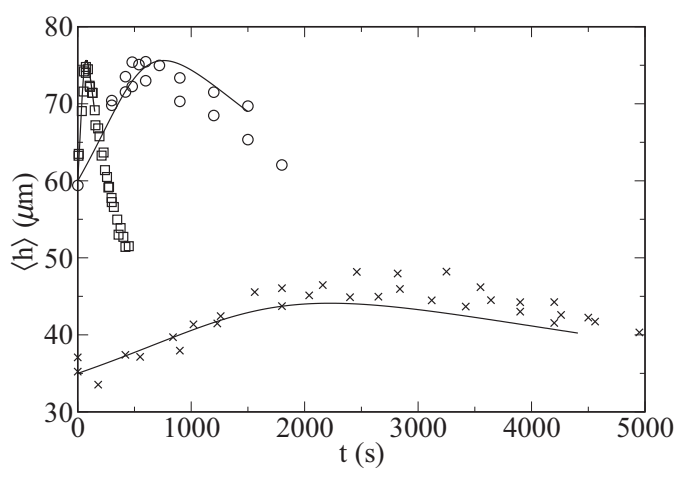

FIG. 3. Thickness evolution for $\theta=5 \pi / 6$. The initial thickness values are $60 \mu \mathrm{m}(\square, \bigcirc)$ and $35 \mu \mathrm{m}(\times)$, with viscosity values of $100 \mathrm{cp}(\square)$ and $1000 \mathrm{cp}(\bigcirc, \times)$. Figure 4 is the dimensionless version of this figure.

and is not taken into account in the analysis. In this way, the analysis is performed only through the mean value of the thickness $\langle h\rangle$ obtained over around 2000 values along $z$. Two different behaviors of the thickness evolution could be observed for positions upstream $(\theta<\pi / 2)$ or downstream $(\theta>\pi / 2)$.

In Fig. 3 the characteristic behavior of the thickness evolution at $\theta=5 \pi / 6$, for different initial thickness and viscosity values, is shown. In all cases, the film thickness reaches a maximum value and then decreases. On the one hand, it can be observed that a film with the same initial thickness evolves more slowly for a more viscous fluid due to the damping effect of viscosity, i.e., the maximum is reached at shorter times if the fluid viscosity is lower. On the other hand, for fluids of equal viscosity, a thicker initial fluid thickness enhances variations since gravity becomes more important. For $\theta=5 \pi / 6$, first (at short time) an increase of the thickness is observed as it receives fluid from the upper part (lower- $\theta$-value sections); later, as liquid is scarce in those upper regions, it begins to drain and therefore the thickness diminishes.

As in previous works [7], the competition between viscous and inertial effects can be considered through a characteristic time $\tau=\frac{\mu R}{\rho g H_{0}^{2}}$. Therefore, the following dimensionless variables were considered: $h=\tilde{h} H_{0}$ for the thickness and $t=\tilde{t} \frac{\mu R}{\rho g H_{0}^{2}}$ for the time. In Fig. 4 a collapse of data from Fig. 3 using these dimensionless variables is presented, showing a universal behavior.

For small values of $\theta(\theta<\pi / 2)$, there is no fluid contribution from upstream zones and a quick decrease of the film thickness is observed, as shown for $\theta=0$ in Fig. 5. The film thickness decreases at a rate that diminishes as $\theta$ increases, which is actually proportional to $\cos (\theta)$, as shown in Eq. (19) in Sec. IV. This rate is approximately zero at $\theta \simeq \pi / 2$, where the input flow from the upper zones and output flow toward lower zones are almost equal, leading to approximately constant thickness at short times (Fig. 6).

\section{B. Unstable region}

At the lower generatrix $(\theta \sim \pi)$, the thickness increases monotonically since there is a net liquid downward flow 


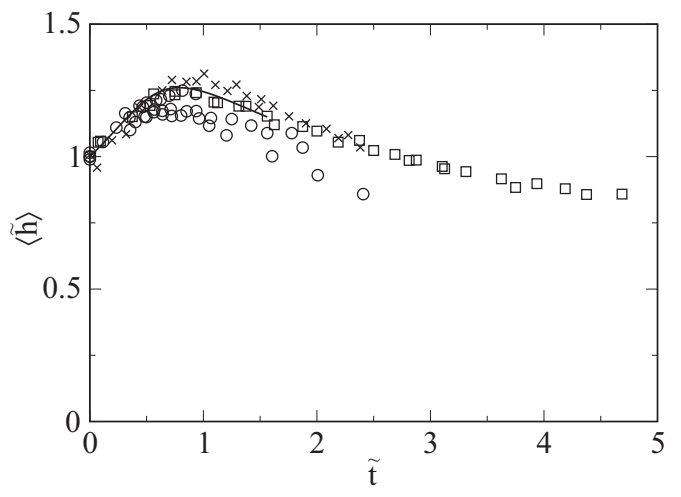

FIG. 4. Evolution of the dimensionless thickness of the film at the $\theta=5 \pi / 6$ generatrix. The markers have the same meaning as in Fig. 3 .

which cumulates there. This fact is clearly shown in Fig. 7, where the dimensionless thickness is plotted as a function of dimensionless time. At the ends of the cylinder, two drops develop before the ones on the center, which eventually grow and fall, but do not move. The effective length of the generatrix is then slightly shorter, but it is still long enough to allow the growth of several drops along it. Afterward, since a dense viscous fluid rests above of a less dense one, a particular type of Rayleigh-Taylor instability is generated [3,5,8-12].

The thickness growth can be followed by ODS measurements until the instabilities start to develop and the sensor must then be separated from the experiment. After that, only video images are available. A line (Fig. 8, upper image) crossing these images at the position where drops eventually appear has been used to study the time evolution of the drops along this surface. The line was followed as a function of time in order to produce a spatiotemporal image, formed by adding horizontal lines for each frame. From this spatiotemporal image, the distance between adjacent drops is measured and the distance distribution is obtained (Fig. 9), leading to a characteristic wavelength $\langle\lambda\rangle=(18 \pm 2) \mathrm{mm}$.

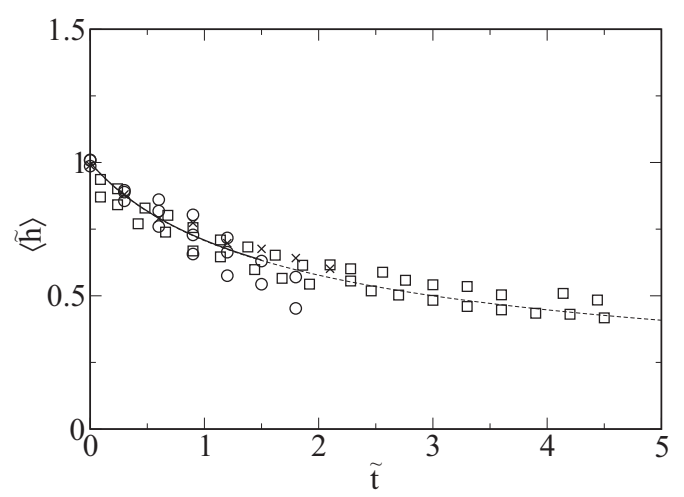

FIG. 5. Evolution of the dimensionless thickness of the film at the $\theta=0$ generatrix. The markers correspond to different initial thickness and fluid viscosity values. The dotted line is given by the dimensionless expression of Eq. (18). The solid line represents numerical integration at short times.

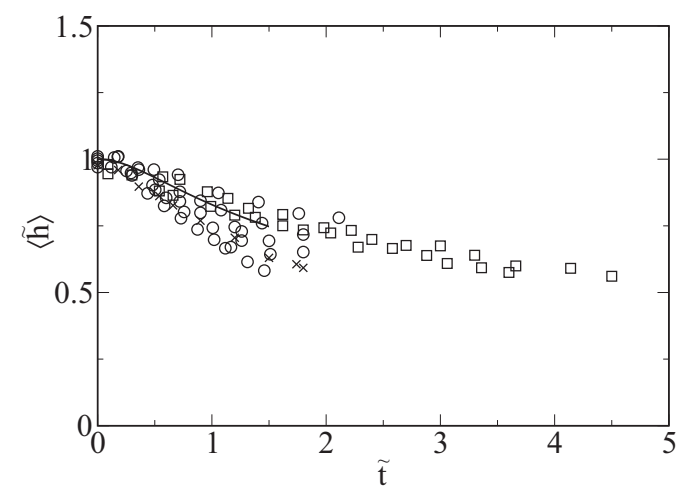

FIG. 6. Evolution of the dimensionless thickness of the film at the $\theta=\pi / 2$ generatrix. The markers correspond to different initial thickness and fluid viscosity values.

\section{THEORETICAL APPROACH}

\section{A. Thickness evolution equation}

An expression for the thickness evolution equation is obtained in the lubrication approximation for the NavierStokes equations in cylindrical coordinates by using very simple boundary conditions. First, since the film thickness $h$ is very small compared to the radius of the cylindrical surface $R$, it is possible to use the lubrication approximation [4]. Using the cylindrical coordinates shown in Fig. 2 with the $\hat{z}$ axis along the cylinder axis, the velocity can be defined as

$$
\bar{V}(r, \theta, z, t)=u(r, \theta, z, t) \hat{\theta}+w(r, \theta, z, t) \hat{z} .
$$

The component along $\hat{r}, v_{r}$, is neglected since $v_{r} \ll u, w$. The velocity, as well as other variables, is defined for $R \leqslant r \leqslant R+$ $h(\theta, z, t)$, where $h(\theta, z, t)$ is the local thickness. Here $H_{0}$ is the (uniform) initial thickness. The cylinder is placed horizontally, so the components $\hat{r}, \hat{\theta}$, and $\hat{z}$ of the Navier-Stokes equation take the form

$$
\begin{gathered}
-\frac{1}{\rho} \frac{\partial P}{\partial r}-g \cos \theta=0 \\
-\frac{1}{\rho R} \frac{\partial P}{\partial \theta}+v \frac{\partial^{2} u}{\partial r^{2}}+g \sin \theta=0
\end{gathered}
$$

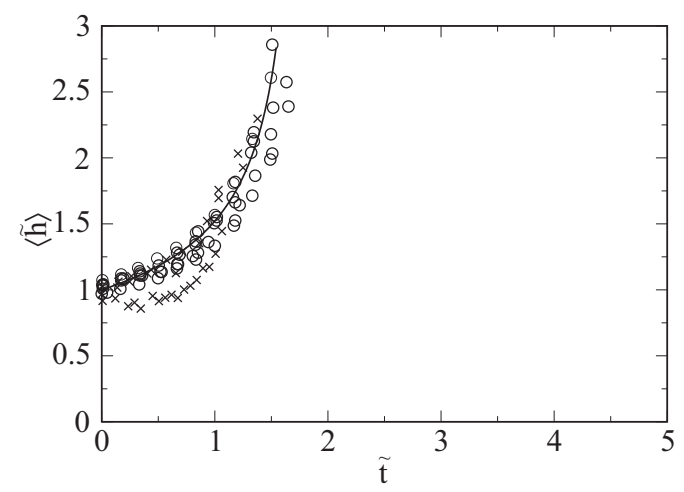

FIG. 7. Evolution of the dimensionless thickness of the film at the $\theta=\pi$ generatrix. The markers correspond to different initial thickness and fluid viscosity values. The solid line is given by the dimensionless expression of Eq. (15). 


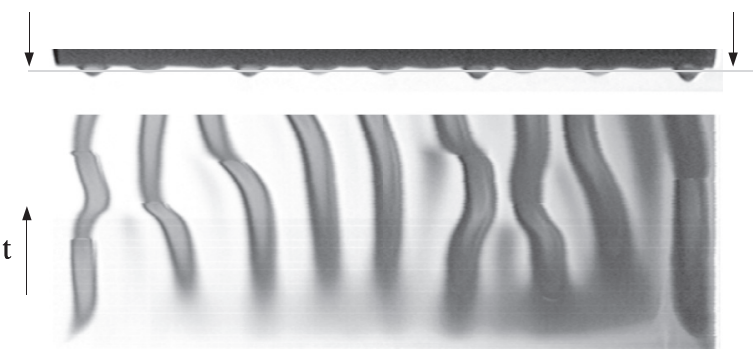

FIG. 8. Typical spatiotemporal diagram obtained by analyzing the evolution of a straight line below the lower generatrix (upper image) where the drops are expected to eventually appear, as a function of time $t$.

$$
-\frac{1}{\rho} \frac{\partial P}{\partial z}+v \frac{\partial^{2} w}{\partial r^{2}}=0
$$

respectively, where $g$ is the gravity, $P$ is the pressure in the liquid, and $v$ and $\rho$ are the viscosity and density, respectively. Equation (2) can be integrated with the free surface boundary condition given by the Laplace pressure

$$
\left.P(r, \theta, z, t)\right|_{r=R+h(\theta, z, t)}=P_{0}-\gamma \mathcal{C}(\theta, z, t),
$$

where $P_{0}$ is the atmospheric pressure, $\gamma$ is the surface tension of the liquid, and $\mathcal{C}(\theta, z, t)$ is the local curvature, which, in this approximation, can be written as $\mathcal{C}(\theta, z, t) \sim \nabla^{2} h(\theta, z, t)$ [13]. Substituting $P$ from (5) in (3) and (4) and using the boundary conditions of no slip in the solid surface and null stress at the free surface,

$$
\begin{gathered}
\left.\bar{V}(r, \theta, z, t)\right|_{r=R}=0, \\
\left.\frac{\partial \bar{V}(r, \theta, z, t)}{\partial r}\right|_{r=R+h(\theta, z, t)}=0,
\end{gathered}
$$

$u(r, \theta, z, t)$ and $w(r, \theta, z, t)$ are obtained. It must be noted that Eq. (7) is an approximation that avoids strong couplings between Eqs. (2)-(4) and implies a simplification coherent with the lubrication approximation.

Since in this approximation only mean velocities across the film are important to the thickness evolution, $u$ and $w$ are averaged between $r=R$ and $R+h(\theta, z, t)$ in order to give the corresponding mean values $U(\theta, z, t)$ and $W(\theta, z, t)$. These

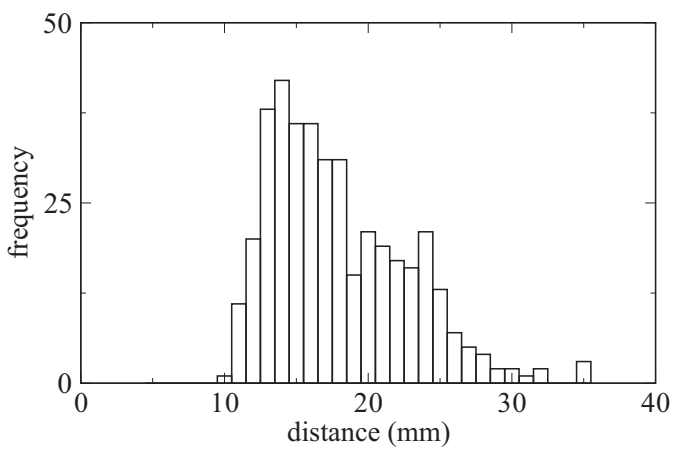

FIG. 9. Distribution of distance between drops (wavelength) obtained from spatiotemporal diagrams, which allows us to determine a characteristic wavelength $\langle\lambda\rangle=(18 \pm 2) \mathrm{mm}$. velocities and the thickness $h$ obey the mass conservation relationship for the film:

$$
\frac{\partial h}{\partial t}=-\nabla \cdot[h(U \hat{\theta}+W \hat{z})] .
$$

Rewriting Eq. (8), the temporal evolution of the thickness $h$ as a function of $\theta$ and $z$ is obtained. The leading terms read

$$
\begin{aligned}
\frac{\partial h}{\partial t}= & -\frac{g h^{2}}{3 v}\left[\frac{3 \sin \theta}{R} \frac{\partial h}{\partial \theta}+\frac{h}{R} \cos \theta\right. \\
& \left.-h \cos \theta \nabla^{2} h+\kappa^{2} h \nabla^{4} h\right]
\end{aligned}
$$

where $\kappa=\sqrt{\gamma / \rho g}$ is the capillary length and $\nabla$ is the twodimensional operator $\left(\frac{1}{R} \frac{\partial}{\partial \theta}, \frac{\partial}{\partial z}\right)$. Equation (9) can be treated in a dimensionless form by taking $h=\tilde{h} H_{0}$ for the thickness and $t=\tilde{t} \frac{\mu R}{\rho g H_{0}^{2}}$ for the time. Additionally, a length scale along $z$ is given by capillary length $\sqrt{\gamma / \rho g}$, which depends only on the properties of the liquid. In the region where the film is stable, there is no reasonable length scale along $z$ and the capillary length is only meaningful around the lower generatrix where instability appears. Equation (9) is integrated numerically at short times, before instabilities appear, using an alternating direction implicit (ADI) scheme [14] with periodic boundary conditions in order to obtain the behavior at the stable region of the film $\left(\theta<170^{\circ}\right)$. The results for every value of $\theta$ experimentally studied $\left(\theta<170^{\circ}\right)$ as well as the variation with the initial thickness are observed to be very close to the experimental results as shown by solid lines in dimensionless form in Figs. 4-7.

\section{B. Linear stability analysis}

In order to obtain the characteristics of the instability that appears at the bottom of the cylinder $(\theta=\pi)$ a linear stability analysis of Eq. (9) is performed, which at $O\left((\theta-\pi)^{0}\right)$ reads

$$
\frac{\partial h}{\partial t}=-\frac{g h^{3}}{3 v}\left[-\frac{1}{R}+\nabla^{2} h+\kappa^{2} \nabla^{4} h\right] .
$$

Standard linear stability analysis gives the following dispersion relation, which is the same as that obtained in previous works for Rayleigh-Taylor instabilities in flat plates $[5,9,12]$ :

$$
\sigma=\frac{g h^{3}}{3 v}\left(q_{\theta}^{2}+q_{z}^{2}\right)\left[\kappa^{2}\left(q_{\theta}^{2}+q_{z}^{2}\right)-1\right] .
$$

Therefore, the maximum of Eq. (11) is obtained for

$$
q=q_{\theta}=q_{z}=\frac{1}{\sqrt{2}} \sqrt{\frac{\rho g}{\gamma}} \rightarrow \lambda=\lambda_{\theta}=\lambda_{z}=2 \pi \sqrt{2} \sqrt{\frac{\gamma}{\rho g}}
$$

and

$$
\sigma_{\max }=\frac{1}{12} \frac{g^{2} h^{3} \rho^{2}}{\mu \gamma}=\frac{1}{\tau_{\min }} .
$$

The expressions in (12) give $\lambda \sim 17 \mathrm{~mm}$. Also considering that the instability appears at $\tilde{t} \sim 1.5$ (Fig. 7), it allows us to obtain an estimate of the critical value of the dimensionless thickness where the instability appears: $\tilde{h} \sim 3$. 


\section{Approximate analytical solutions of Eq. (9)}

\section{Approximation at $\theta \sim \pi$}

Equation (10) is an approximation of Eq. (9). A further approximation, neglecting the spatial derivatives of $h$, leads to an integrable equation

$$
\frac{\partial h}{\partial t}=\frac{1}{R} \frac{g}{3 v} h^{3},
$$

which gives

$$
h_{\pi}(t)=\sqrt{\frac{3 R v H_{0}^{2}}{3 R v-2 g H_{0}^{2} t}} .
$$

This expression is valid for

$$
t<\frac{3 R v}{2 g H_{0}^{2}},
$$

that is, for $\tilde{t}<1.5$. The numerical simulations and the prediction of relation (15) are in excellent agreement and this expression is plotted in Fig. 7 as a solid line.

\section{Approximation at $\theta \sim 0$}

The same procedure applied to Eq. (9) can be applied for the upper generatrix; the following equation is obtained:

$$
\frac{d h}{d t}=-\frac{1}{R} \frac{g}{3 v} h^{3},
$$

which gives

$$
h_{0}(t)=\sqrt{\frac{3 R v H_{0}^{2}}{3 R v+2 g H_{0}^{2} t}} .
$$

Equation (18) is valid for any $t>0$ and gives the right behavior even for long times since $h_{0} \rightarrow 0$ for $t \rightarrow \infty$. Also, in this case, the agreement between the numerical simulations and relation (18) is excellent. In Fig. 5, this expression is plotted as a dotted line while the numerical result (valid for shorter times) is plotted as a solid line.

\section{Approximation to initial rate $\partial \mathrm{h} / \partial t$}

Another useful approximation can be obtained by taking the same approximation as before but keeping the dependence on $\theta$. From Eq. (9) we have

$$
\left.\frac{\partial h}{\partial t}\right|_{t=0^{+}}=-\frac{g h^{3}}{3 v R} \cos (\theta),
$$

which reproduces well the initial behavior of the thickness.

\section{ANALYSIS AND DISCUSSION}

Systematic experiments on the flow around a horizontal cylinder initially coated with a liquid film of uniform thickness have been performed. The results show that the film thickness evolution can be separated into two regimes with stable and unstable behavior, the latter being almost confined to the lower generatrix. On the upper half of the cylinder $(\theta \leqslant \pi / 2)$ a stable behavior is observed during the whole experiment and the film thickness only decreases monotonically with time: The liquid gradually flows toward the lower part of the cylinder. On the lower half of the cylinder $(\theta>\pi / 2)$ the film's local thickness is stable during the whole experiment except near $\theta \sim \pi$. For $\pi / 2<\theta \lesssim \pi$ the film's local thickness increases to a maximum due to the flow of liquid from upper zones. Afterward, when this flow diminishes, the film thickness decreases monotonically with time. For $\theta \sim \pi$, where the instability eventually appears, the local thickness increases monotonically until it destabilizes.

Experiments show that in the unstable region $(\theta \lesssim \pi)$, after an initial stable growth, the film destabilizes along the axis of the cylinder, originating drops, almost all of which start to exponentially grow at the same time. This behavior may be due to the fact that the characteristic time of the instability growth $1 / \sigma$ strongly depends on the local thickness that is, at the same time, growing because of the flow from upper generatrices. When the thickness attains a critical value, the instability can grow fast enough and then become observable. This relationship is still under study.

After instabilities appear, as shown in Fig. 8, drops start to move, sometimes coalesce, and eventually fall. This behavior cannot be assigned to any irregularity on the local generatrix, since it is different from one experiment to another, or to the horizontality of the experiment, since no preferential direction is observed in the displacements.

The differential equation of the thickness evolution in two dimensions ( $\theta$ and $z$ of cylindrical coordinates) using the lubrication approximation has been obtained. This equation (9) was found to be, as is typical in thin film flow problems [7], nonlinear and fourth order in both cylindrical coordinates. It has been integrated numerically for short times using an ADI scheme [14] and excellent agreement with experimental results was found. Also, analytical solutions can be obtained for $\theta=$ $\pi$ and 0 at very short times (in the stable regime). The agreement between the analytical solution from Eqs. (15) and (18) and the numerical integration is excellent. For $\theta=0$, Eq. (15) remains valid for long times describing the experimental behavior (Fig. 5): The thickness decreases monotonically.

It is interesting to compare Eq. (9) and the corresponding equation from Ref. [5], which we reproduce here for clarity as Eq. (20). Both equations are valid for the same kind of condition-a horizontal substrate coated with a liquid film that is hanging above the surrounding air-but, while in the case of Ref. [5] there is no incoming flow, in this work the local thickness increases due to the flow the film receives from above. A possible consequence of the incoming flow might be observed in the behavior of the instability, which is drastically different in each case: In the cylinder, the drops move randomly and sometimes coalesce, while in the beam (20), drops grow and eventually fall, but without any displacement. Nevertheless, its origin remains unclear:

$$
\frac{\partial h}{\partial t}=-\frac{g h^{3}}{3 v}\left[\nabla^{2} h+\kappa^{2} \nabla^{4} h\right] .
$$

The difference between these equations is the presence of the first term on the right-hand side of Eq. (9), which takes into account the thickness growth at the bottom generatrix [see Eq. (15)]. This term is constant and therefore does not contribute to the linear stability analysis. In this way, both equations give the same dispersion relation and therefore the same expected wavelength. 
Effectively, a linear stability analysis [15] was performed at the lower generatrix where an instability was expected to develop. The wavelength of fastest growth and the maximum of the growth rate were obtained for a wavelength close to that of the Rayleigh-Taylor instability. Experimentally, even if drops move and measurements depend on time, distances can be obtained and therefore wavelengths can be determined (Fig. 9): $\langle\lambda\rangle=(18 \pm 2) \mathrm{mm}$, which reasonably agrees with the prediction for the wavelength of fastest growth $\lambda \sim 17 \mathrm{~mm}$, which, as explained above, is the same as that in [5].

\section{ACKNOWLEDGMENTS}

The authors thank CONICET and University of Buenos Aires for financially supporting this work.
[1] O. Kirk, Encyclopedia of Chemical Technology (Wiley, New York, 1998), Vol. 6, pp. 595-716.

[2] L. Schwartz and D. E. Weidner, J. Eng. Math. 29, 91 (1995).

[3] D. E. Weidner, L. Schwartz, and M. H. Eres, J. Colloid Interface Sci. 187, 243 (1997).

[4] D. J. Acheson, Elementary Fluid Dynamics (Oxford University Press, New York, 1994), pp. 238-259.

[5] M. Lenschen, A. Calvo, and M. Cachile, Phys. Rev. E 81, 017302 (2010).

[6] Acuity Research: http://www.acuitylaser.com.

[7] L. Kondic, SIAM Rev. 45, 95 (2003).

[8] S. G. Yiantsios and B. G. Higgins, Phys. Fluids A 1, 1484 (1989).

[9] P.-G. de Gennes, F. Brochard-Wyart, and D. Quéré, Gouttes, Bulles, Perles et Ondes (Belin, Paris, 2002).
[10] J. P. Kizito, F. B. Weng, Y. Yatomani, and S. Ostrach, Proceedings of the Fourth Microgravity Fluid Physics and Transport Phenomena Conference, Cleveland, 1998 (National Center for Microgravity Research on Fluids and Combustion, Cleveland, 1999), pp. 21-23.

[11] J. R. de Bruyn, Phys. Fluids 9, 1599 (1997).

[12] M. Fermigier, L. Limat, J. E. Wesfreid, P. Boudinet, and C. Quillet, J. Fluid Mech. 236, 349 (1992).

[13] S. O'Brien and L. Schwartz, Encyclopedia of Surface and Colloid Science (Dekker, New York, 2001), pp. 5283-5297.

[14] T. P. Witelski and M. Bowen, Appl. Numer. Math. 45, 331 (2003).

[15] S. Chandrasekhar, Hydrodynamics and Hydromagnetic Stability (Dover, New York, 1961), Chap. X, pp. 428-480. 